\title{
Genitourinary tuberculosis in North America: A rare clinical entity
}

\author{
Michael W. Sourial, MD; ${ }_{i}^{*}$ Fadi Brimo, MD; ${ }^{\dagger}$ Ruth Horn, MD; ${ }^{;}$Sero Andonian, MD, MSc, FRCSC, FACS
}

*Division of Urology, Department of Surgery, McGill University Health Centre, Montreal, QC; †Department of Pathology, McGill University Health Centre, Montreal, QC; §Department of Microbiology, McGill University, and Division of Infectious Diseases, McGill University Health Centre, Montreal, QC

Cite as: Can Urol Assoc J 2015;9(7-8):E484-9. http://dx.doi.org/10.5489/cuaj.2643 Published online July 17, 2015.

\section{Abstract}

Introduction: Although tuberculosis (TB) is the most common cause of mortality from infectious diseases worldwide, genitourinary TB in North America is rare. We review 3 cases of genitourinary TB diagnosed within the last 5 years.

Cases: The first case is that of a 76-year-old African-Canadian woman who was referred for percutaneous nephrolithotomy of right lower pole renal stones. Although renal TB was suspected, her initial urinary TB culture was negative. On follow-up imaging, she developed bilateral ureteral thickening and ureteroscopic biopsy confirmed necrotizing granulomata. Repeat urine cultures were positive for M. tuberculosis. The second case is a 73-yearold Italian-Canadian woman who was referred for ureteroscopic biopsy of left thickened ureter to rule out urothelial carcinoma. Initial urine TB cultures were negative, despite biopsies confirming granulomatous inflammation. She was closely followed with urine cytologies and TB cultures. Repeat urine culture was positive for M. tuberculosis. Both patients were treated with a course of anti-tuberculous agents and indwelling ureteral stents to relieve ureteral obstruction. The third case is a 70-year-old Cree woman who was referred for percutaneous nephrolithotomy of a left "staghorn stone" in an atrophic left kidney. Thirty years earlier she had been treated for pulmonary TB in addition to ileocystoplasty for a "thimble" bladder. A computed tomography scan showed autonephrectomized left kidney. Her urine TB cultures were negative. She was placed on prophylactic antibiotics for her recurrent bacterial urinary tract infections.

Conclusion: Genitourinary TB may present in various subtle ways, and the astute clinician must have a high index of suspicion for this disease in patients with atypical clinical and radiologic findings. In addition, TB urine cultures should be repeated when there is high index of suspicion.

\section{Introduction}

Tuberculosis (TB) is the most common cause of mortality from infectious disease worldwide, with about $95 \%$ of cases occurring in developing countries. ${ }^{1}$ Genitourinary TB is the third most common form of extrapulmonary TB after pleural TB and lymphatic TB in North America., 2,3 Patients have non-specific symptoms and atypical presentations, which often lead to difficulty and delay in diagnosis. Urinalysis usually shows culture-negative pyuria and hematuria. A special acid-fast bacterial culture is required, taking up to 8 weeks for Mycobacterium tuberculosis to grow, with a false negative rate as high as $20 \% .{ }^{4,5}$ Imaging findings are also non-specific and may even further delay diagnosis. ${ }^{6}$ The astute clinician must have a high degree of suspicion for genitourinary TB in patients presenting with non-specific symptoms, culture-negative pyuria, and for whom imaging studies seem atypical. We present 3 cases of genitourinary TB clinically diagnosed by a urologist within the last 5 years; these cases remind clinicians of this rare but existing clinical condition in North America.

\section{Case 1}

A 76-year-old African-Canadian woman initially presented to a community urologist with dysuria and complex calcifications at the lower pole of the right kidney (Fig. 1a, Fig. 1b). The patient was referred to our tertiary care centre for percutaneous nephrolithotomy (PCNL) of the right renal stones. Due to the unusual features, magnetic resonance imaging was performed to further characterize the calcified cystic lesion (Fig. 1c). Initial differential diagnoses included partial staghorn stone, calyceal diverticulum with stones and chronic pyelonephritis, cystic renal cell carcinoma with calcification, TB, and fungus balls. Initially, the patient denied TB exposure. Lasix renal scan showed that the right kidney contributed 22\% with an overall effective renal plasma flow (ERPF) of $420 \mathrm{~mL} /$ min. There was no obstruction bilaterally. Urine cytology, TB, and fungal cultures were sent and were all negative. Repeat imaging with triphasic CT urogram 18 months after the initial presentation showed bilateral ureteral thickening with new left moderate hydroureteronephrosis (Fig. 1d). The repeat lasix renal scan showed reduced global renal function with an ERPF 
of $303 \mathrm{~mL} / \mathrm{min}$ and right kidney contributing $21 \%$. In addition, there was bilateral partial obstruction with $\mathrm{T}_{1 / 2}$ of 30 minutes for the left side, and 42 minutes for the right side. The patient underwent cystoscopy, which revealed a "golf-hole" right ureteral orifice. Bilateral retrograde pyelogram confirmed bilateral ureteral obstructions with severely distorted architecture of the right renal calyces. Bilateral ureteroscopy with biopsies of the inflammatory areas showed necrotizing granulomas in both the right renal pelvis and distal left ureter. There were no signs of urothelial carcinoma. Grocott stains were negative, but Ziehl-Neelsen stains showed a large number of acid fast bacilli (Fig. 1e, Fig. 1f). Repeat TB urine culture was positive for M. tuberculosis sensitive to all agents. The patient was referred to the infectious diseases clinic and was treated with a standard 6-month regimen of anti-tuberculous therapy (4 months of isoniazid, rifampin, and pyrazinamide and an additional 2 months of isoniazid and rifampin). Furthermore, she had bilateral indwelling ureteral stents inserted and changed at routine intervals. Follow-up TB cultures were negative after she finished her course of anti-tuberculous therapy. Once the TB cultures were positive, the patient acknowledged that her grandfather had TB and she was exposed to TB as a child in Canada.

\section{Case 2}

A 73-year-old Italian-Canadian woman initially presented to a community urologist with gross hematuria and early

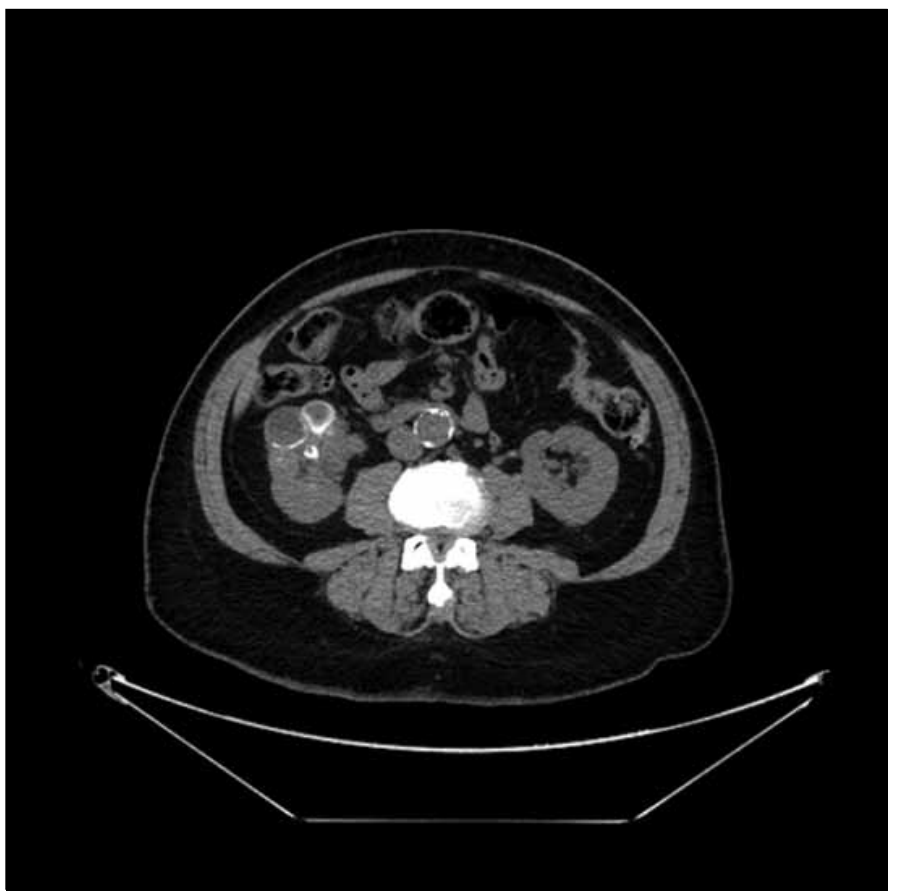

Fig. 1a. Non-contrast computed tomography scan (axial image) showing multiple large areas of calyceal dilatation with cortical thickening and peripheral calcification of the lower pole of the right kidney. morning urgency. Triphasic CT urogram showed left ureteral thickening with an atrophic left kidney (Fig. 2a). Her medical history was significant for several malignancies, including breast cancer for which she had received neoadjuvant 5-fluorouracil, etoposide, cyclophosphamide, and docetaxel prior to left modified radical mastectomy with axillary lymph node dissection; laryngeal cancer treated with chemotherapy and radiation; and cholangiocarcinoma treated with Whipple's procedure followed by radiation therapy. The patient was referred for left ureteroscopy with biopsies to rule out urothelial carcinoma. Endoscopically, the whole left collecting system was inflamed with fluffy exudative material. Left ureteral biopsies showed inflammatory exudates with necrosis and cells suggestive of a necrotizing granulomatous inflammation. There was no evidence of urothelial carcinoma. The diagnosis of genitourinary TB was considered. However, Ziehl-Neelsen stains were negative for acid fast bacilli (Fig. 2b). In addition, her urine cytologies, fungal and TB cultures were negative. She was followed with serial urine cytology, TB cultures, and axial imaging. Follow-up CT urography 2 years later showed development of a new right-sided hydronephrosis in a solitary functioning right kidney, with a rising serum creatinine from 70 to $118 \mu \mathrm{moL} / \mathrm{L}$. Lasix renal scan confirmed highgrade obstruction from the solitary-functioning right kidney. Cystoscopy showed a "golf-hole" right ureteral orifice. Right retrograde pyelography showed a dilated distal ureter with right ureteropelvic junction obstruction. In addition, right

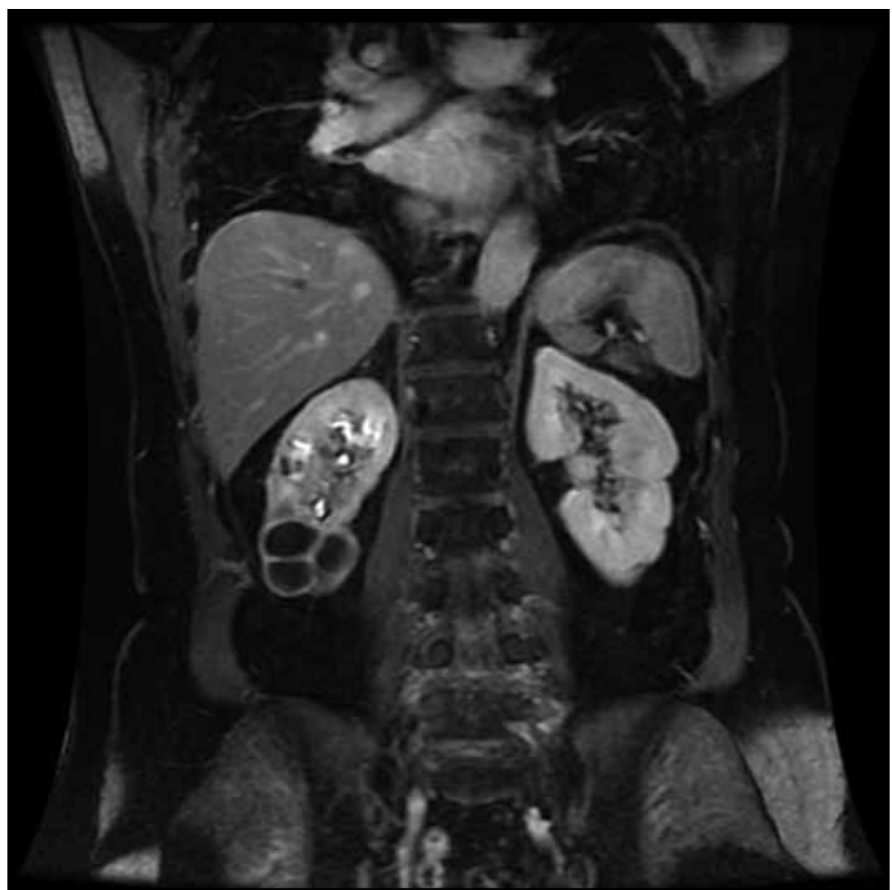

Fig. 1b. Coronal magnetic resonance image showing chronic calyceal dilatation with cortical thickening and heterogeneous enhancement of the lower pole of the right kidney. 


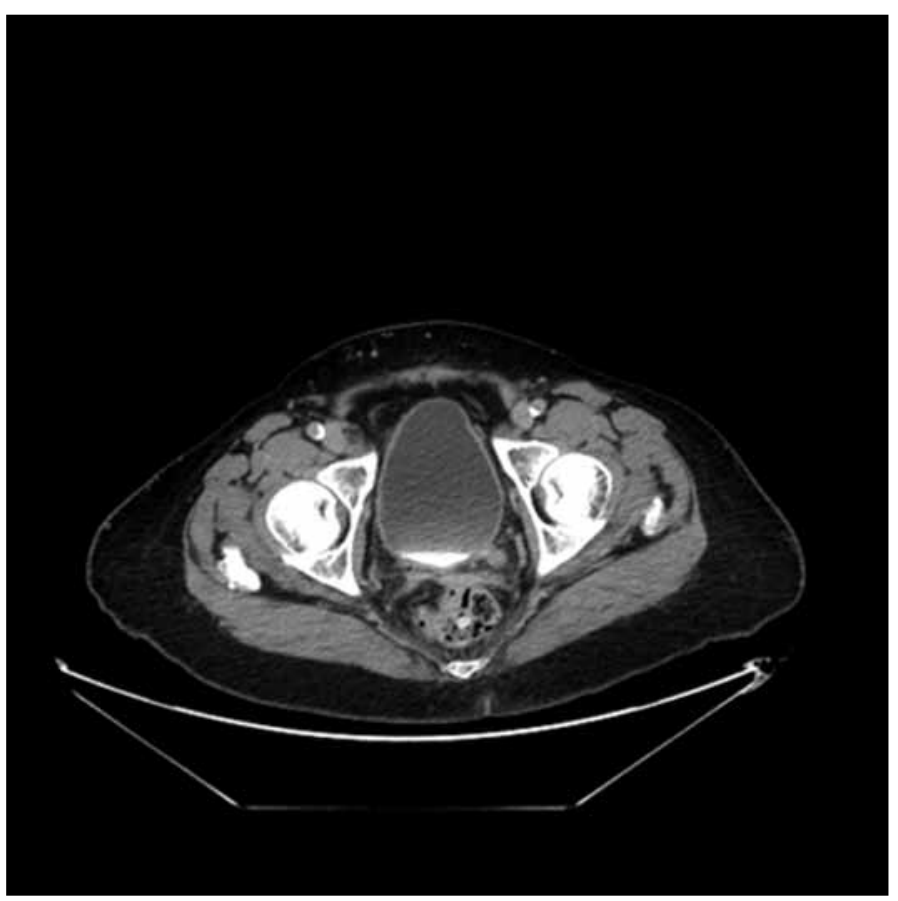

Fig. 1c. Delayed image of triphasic computed tomography scan showing left distal ureteral thickening.

ureteroscopy showed inflamed tight ureteropelvic junction. Biopsies confirmed the absence of urothelial carcinoma, but failed to show granulomata. The patient was managed with chronic right indwelling ureteral stents. Repeat urine TB culture was positive for $M$. tuberculosis sensitive to standard agents. The patient was referred to the infectious diseases clinic and was treated with a standard 6-month regimen of anti-tuberculous therapy (2 months of isoniazid, rifampin, and pyrazinamide and an additional 4 months of isoniazid and rifampin). After finishing her course of anti-tuberculous

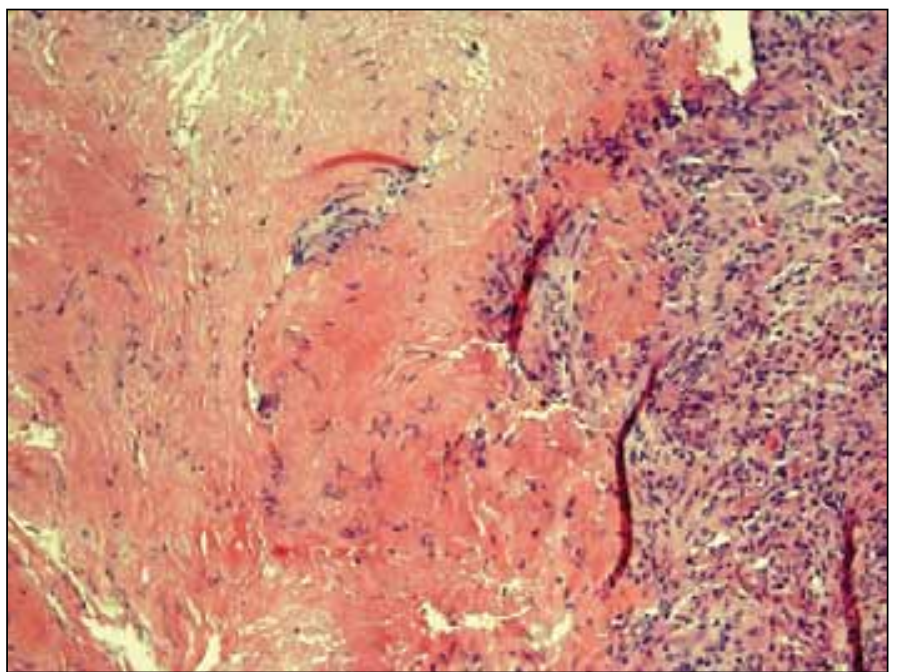

Fig. 1e. Right renal pelvis biopsy showing collection of epithelioid histiocytes on the right associated with caseating-type necrosis on the left

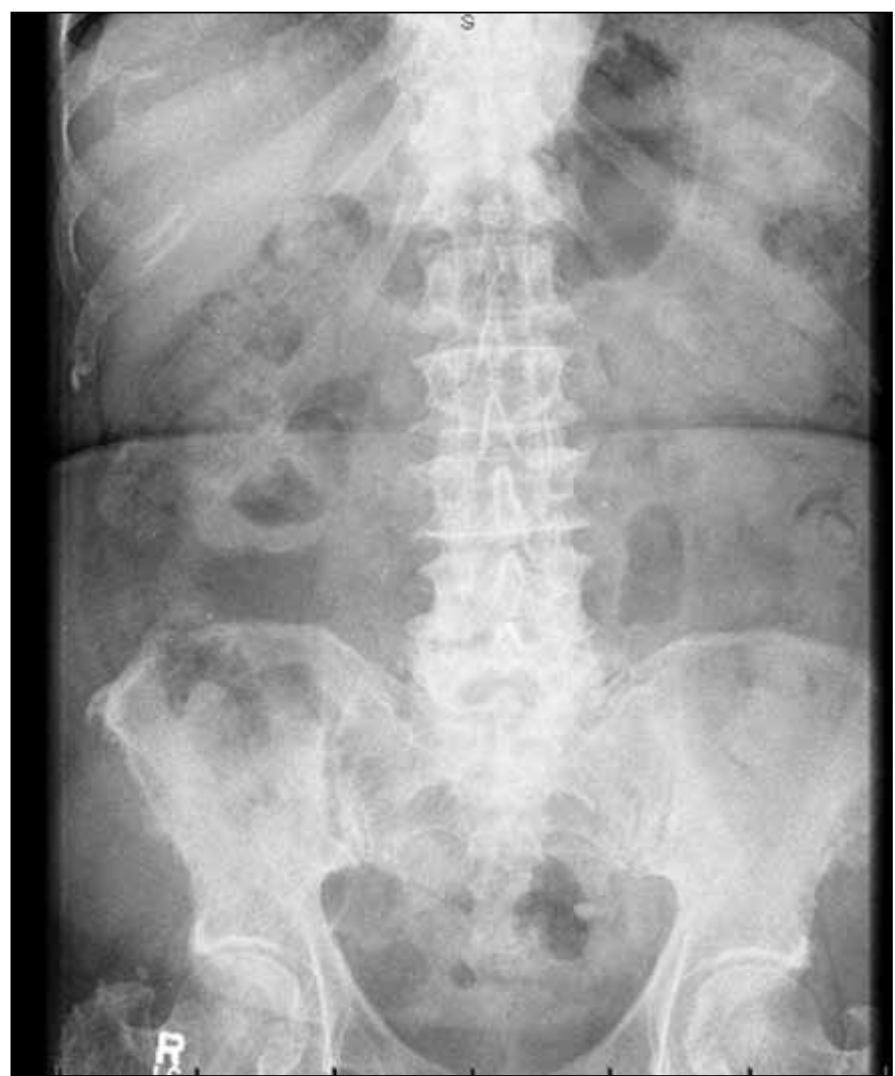

Fig. 1d. Initial plain kidney-ureter-bladder radiograph. The right renal calcifications are not well appreciated.

therapy, urinary TB cultures were negative and her earlymorning urgency improved. She continues to be managed conservatively with chronic right indwelling ureteral stent changes. She denies exposure to TB.

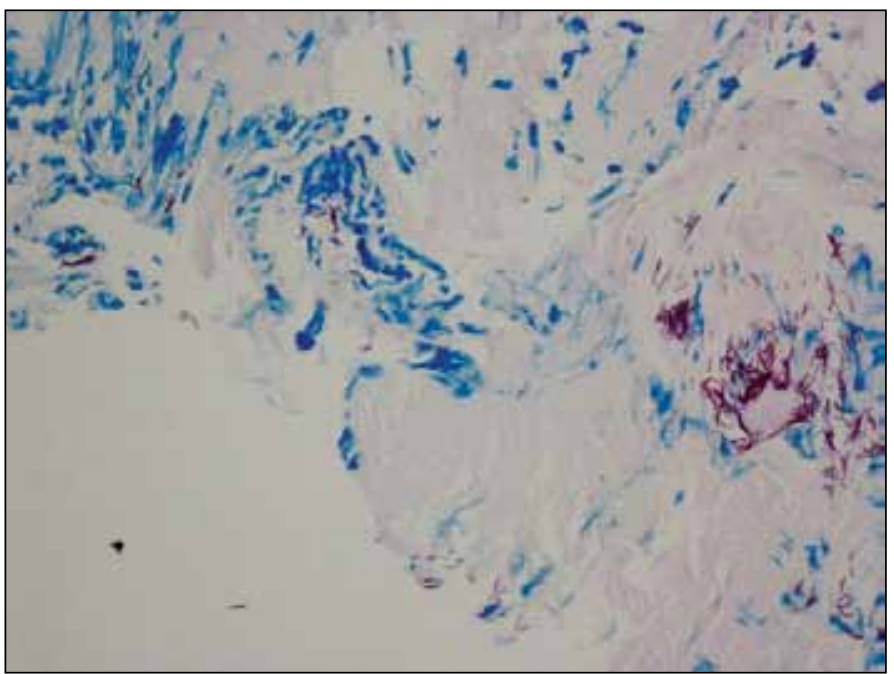

Fig. 1f. Zeihl-Neelson staining showing numerous acid fast bacilli (red colour). 


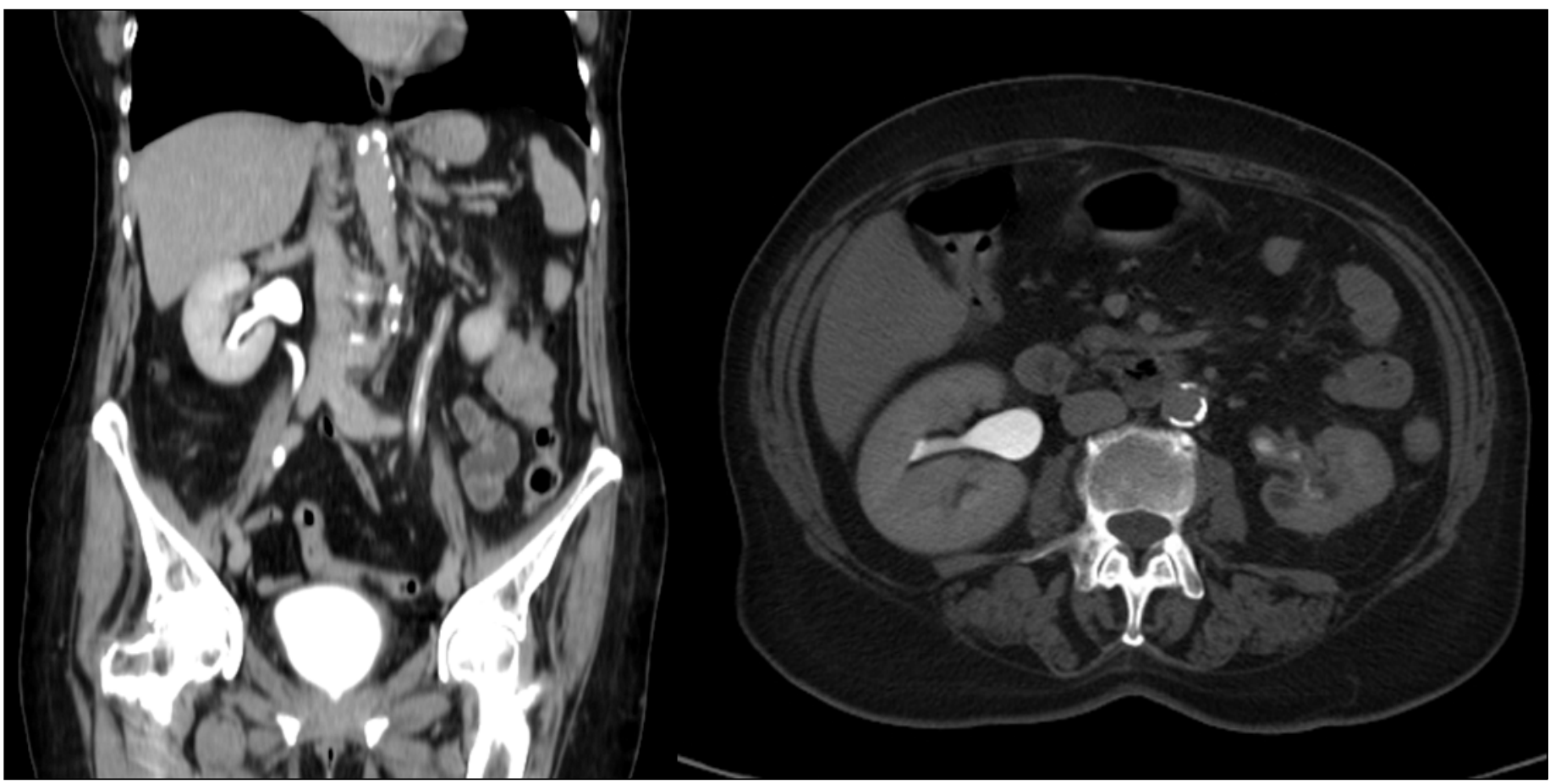

Fig. 2a. Axial and coronal delayed phase computed tomography scan showing marked thickening of the left proximal ureter and renal pelvis, with right moderate hydronephrosis.

\section{Case 3}

This 70-year-old diabetic Cree woman presented to a community urologist with chronic recurrent bacterial urinary tract infections and was referred to our tertiary stone centre for PCNL of her "staghorn stone" in the left atrophic kidney. A review of her medical records showed that she was treated for pulmonary TB at the age of 40, with a 1-year course of isoniazid, ethambutol, and streptomycin, and was kept

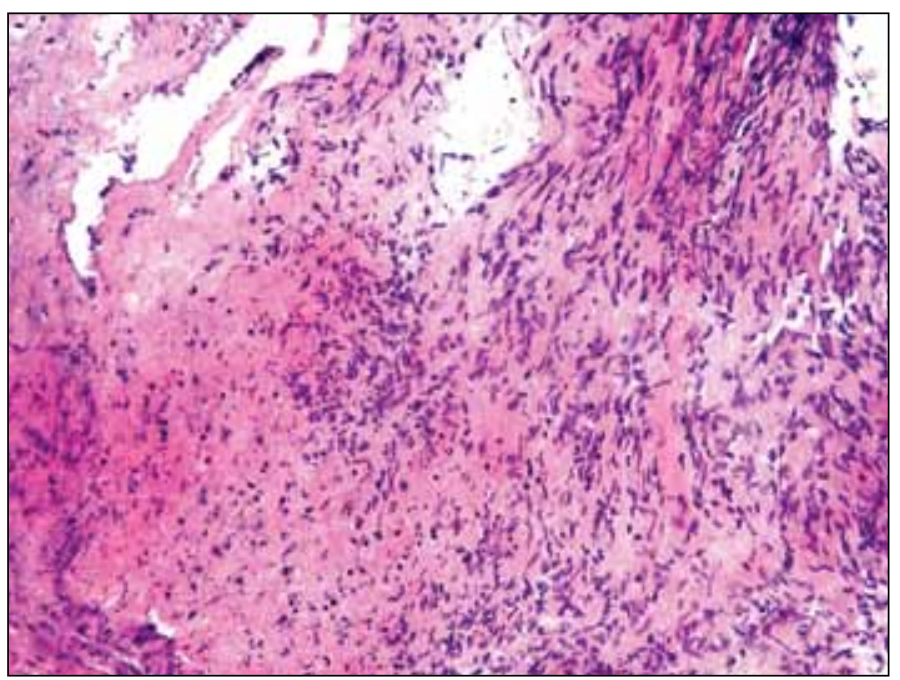

Fig. $\mathbf{2 b}$. Left ureteral biopsy showing necrotizing granulomatous inflammation in a sanitarium for some time. In addition, she had undergone augmentation ileocystoplasty for "thimble" bladder. Urine TB cultures were negative. Axial imaging showed a heavily calcified, autonephrectomized left kidney or putty kidney (Fig. 3). Since her urine TB cultures were negative, she did not require further anti-tuberculous therapy. She was treated conservatively with prophylactic oral antibiotics for her recurrent urinary tract infections and was advised to continue timed voiding.

\section{Discussion}

TB is the most common cause of mortality from infectious disease worldwide, with about $95 \%$ of cases occurring in developing countries. ${ }^{1}$ Genitourinary TB is the third most common form of extrapulmonary TB after pleural TB and lymphatic TB in several countries, including Canada and the United States. ${ }^{2,3}$ According to the Centers for Disease Control and Prevention, the number of TB cases reported and the case rate is decreasing, with a rate of 3 cases per 100000 people in the United States in 2013. ${ }^{7}$ Immunocompromised patients, particularly HIV patients, are more at risk of developing TB. While systemic symptoms are less common, patients with genitourinary TB may present with hematuria, flank pain, or most commonly with non-specific urinary symptoms. This is the reason why TB is labelled as the "Great Imitator." In addition to all 3 patients presenting 


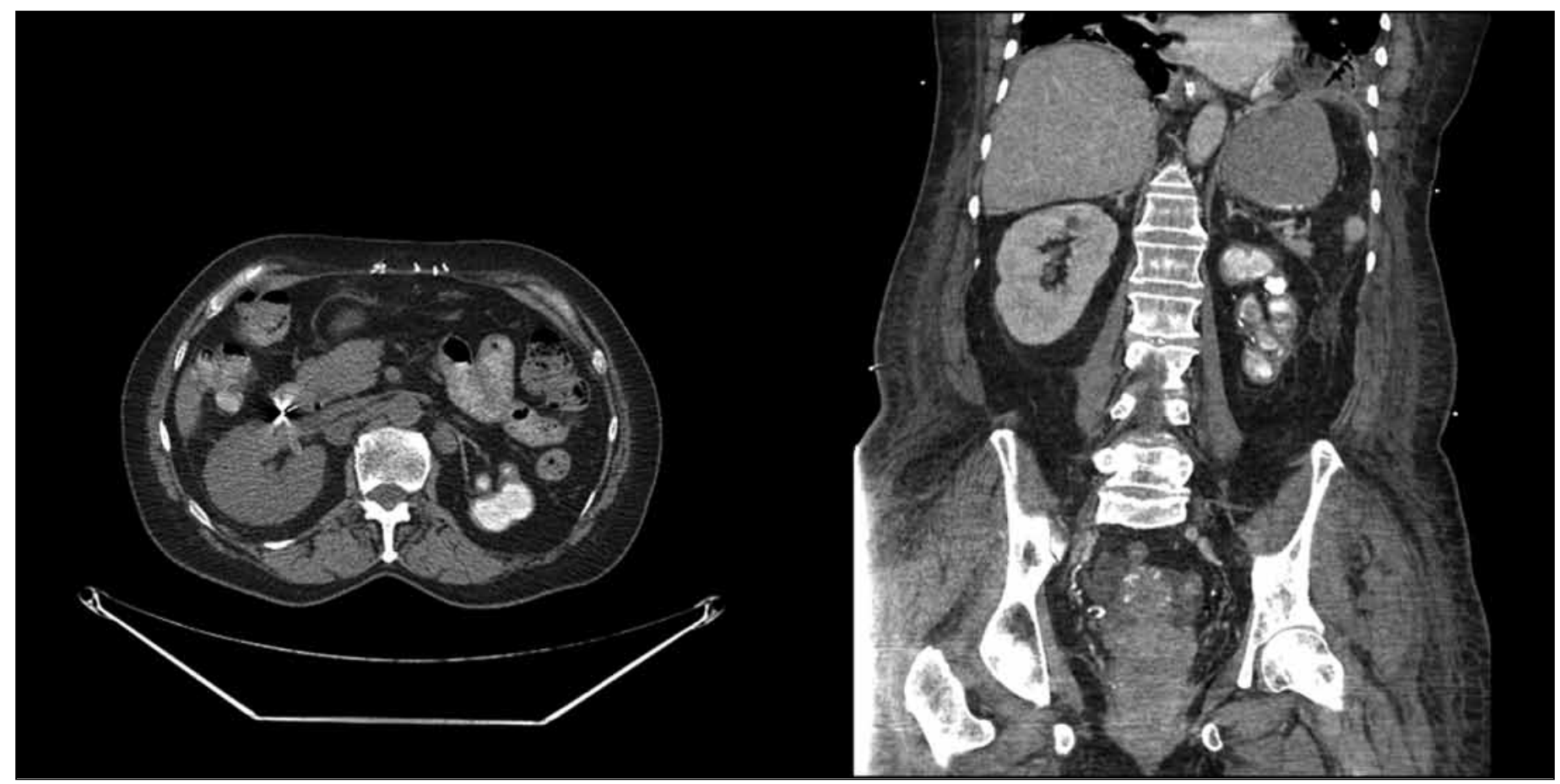

Fig. 3. Axial and coronal non-contrast computed tomography scan showing an atrophic left kidney with severe calcifications (autonephrectomized left kidney or putty kidney).

with non-specific lower urinary tract symptoms, the first and third patients had renal calcifications and were referred for PCNL, while the first 2 patients had ureteral thickening and were referred for ureteroscopic biopsies to rule out urothelial carcinoma.

Renal TB results from hematogenous spread of the tubercle bacilli, which lodge in the corticomedullary junction and form cortical granulomas because of the high blood perfusion rate and blood oxygen saturation..$^{1,6}$ This is why renal involvement is the most affected organ in genitourinary TB. In immune-competent patients, these granulomas may stay latent for many years. With immune suppression, mycobacteria are reactivated and are spilled into the nephron and get trapped within the narrow loop of Henle in the papilla causing caseating papillary lesions. ${ }^{4}$ This is when bacilli are shed into the urine spreading TB antegradely to the urothelium of the renal pelvis, ureter, bladder and adjacent genital organs. ${ }^{4}$ Often, the involved organs may be irreversibly damaged at the time of presentation as was seen in the present series where all 3 patients had renal atrophy at the time of diagnosis of genitourinary TB. In addition, the first 2 patients had ureteral involvement.

In over $90 \%$ of genitourinary TB, there is an abnormal urinalysis, with the most common finding being sterile pyuria in an acidic urine with or without hematuria. ${ }^{8} \mathrm{~A}$ special acidfast stain and mycobacterial culture are required to identify Mycobacterium tuberculosis. However, the culture takes 6 to 8 weeks to grow and it has a false negative rate as high as $20 \% .{ }^{4,5}$ Therefore, at least 3 first morning midstream urine samples are advised to isolate the organism. ${ }^{6,8}$ In addition, patients need to be off antibiotics at the time of urine collection since antibiotics may inhibit mycobacterial growth in culture. ${ }^{8}$ Patients with pulmonary or disseminated TB may also have an incidentally positive urine culture for M. tuberculosis without genitourinary involvement. ${ }^{8}$ Unfortunately, for the first 2 patients, the initial urine TB cultures were negative and may have delayed the initiation of the antituberculous therapy.

Tri-phasic CT remains the mainstay imaging study for cross-sectional imaging in renal TB, which can easily detect uneven caliectasis, calcifications, or urothelial thickening. ${ }^{5}$ Although these findings may be "typical" of GU TB, the differential diagnoses include partial staghorn stone, calyceal diverticulum with stones, chronic pyelonephritis, cystic renal cell carcinoma with calcification, fungal infections and urothelial carcinoma. Chest imaging may be abnormal in $40 \%$ to $75 \%$ of patients with genitourinary TB. However, these abnormalities may be due to past tuberculosis and may not necessarily indicate active disease. ${ }^{8}$

A paucity of literature exists for the treatment of genitourinary TB. The Centers for Disease Control and Prevention suggests treating renal TB primarily with medical therapy, and a 6-month regimen is recommended. ${ }^{9}$ Recommendations include 2 months of isoniazid, rifampin, pyrazinamide, and ethambutol, followed by 4 months of isoniazid and rifampin, unless the organism is resistant to first-line drugs. ${ }^{9}$ In the pres- 
ent series, the first 2 patients with re-activated genitourinary TB were treated with a 6-month course of anti-tuberculous therapy. However, they did not receive ethambutol due to renal insufficiency and the fact that their $M$. tuberculosis was pan-sensitive. The last patient did not require further anti-tuberculous therapy since she presented with end-stage TB of her left kidney. She had previously been treated with a 1-year course of isoniazid, ethambutol, and streptomycin for pulmonary TB 30 years earlier.

Extirpative surgical management was the main treatment modality to reduce mortality in patients with renal TB prior to the discovery of anti-tuberculous medications. ${ }^{6}$ Although some authors still advocate nephrectomy for end-stage renal TB to prevent latent tuberculous reactivation, most advocate medical therapy and nephrectomy only in patients with persistent hypertension or flank pain in a non-functioning kidney. ${ }^{6,9}$ According to the most recent European Association of Urology Guidelines for the management of genitourinary TB, nephrectomy is indicated in (1) non-functional renal units with or without calcification; (2) extensive disease involving the whole kidney, together with hypertension and ureteropelvic junction obstruction; and (3) coexisting renal carcinoma. Nephrectomy is also indicated in cases of complications, such as severe urinary tract infection, stones, and/or hypertension due to tuberculous nephropathy. Interestingly, $50 \%$ of nephrectomy tissue may still show active M. tuberculosis despite post-chemotherapy sterile urine. It is recommended that patients undergo radical surgery within the first 2 months of intensive chemotherapy. ${ }^{10}$

Ureteral obstruction is treated with stents or nephrostomy tubes as clinically indicated. The use of corticosteroids in addition to stenting for ureteral obstruction is discussed in the literature, but its efficacy in this setting remains unclear. According to the latest Center for Disease Control guidelines, corticosteroids are not recommended for the treatment of genitourinary TB. ${ }^{9}$ Response to treatment may be difficult to assess, and should be based on clinical and radiologic findings, and eradication of the mycobacterium on subsequent cultures. ${ }^{9}$ Given the advanced age and multiple comorbidities of all 3 patients presented in this series, conservative urologic management, rather than extirpative or reconstructive surgical managment, was followed chronic indwelling ureteral stent changes for the first 2 patients and prophylactic antibiotics for the last patient with recurrent bacterial urinary tract infections.

There are several take-home messages from these 3 cases. First, genitourinary TB may present in subtle ways, and clinicians must have a high index of suspicion in patients presenting with atypical clinical and radiologic findings. Second, there is still taboo regarding TB exposure and patients may not feel comfortable disclosing their previous
TB exposures as seen in the first case. Third, since there is a high false negative rate of $20 \%$, one negative mycobacterial urine culture does not mean the absence of genitourinary TB. At least 3 first morning midstream urine samples are advised to isolate the organism. Finally, a multidisciplinary approach with infectious disease and urology teams is crucial to providing optimal patient care, with anti-tuberculous medications remaining the cornerstone of treatment, and surgical management reserved for specific indications.

\section{Conclusion}

Genitourinary TB is a rare but existing clinical entity in North America. This diagnosis should be entertained in patients presenting with atypical clinical and radiological findings. In addition, diagnosis of genitourinary TB should not be excluded with the first negative urine TB cultures. Patients should be referred to infectious diseases specialists for further urinary TB testing and anti-tuberculous therapy.

Competing interests: The authors declare no competing financial or personal interests.

This paper has been peer-reviewed.

\section{References}

1. Muttarak M, ChiangMai WN, Lojanapiwat B. Tuberculosis of the genitourinary tract: Imaging features with pathological correlation. Singapore Med J 2005;46:568-74.

2. Daher ED, da Silva Jr GB, Barros EJG. Renal tuberculosis in the modern era. Am J Trop Med Hyg 2013;88:54-64. http://dx.doi.org/10.4269/ajtmh.2013.12-0413

3. Kulchavenya E. Extrapulmonary tuberculosis: Are statistical reports accurate? Ther Adv Infect Dis 2014;2:61-70. http://dx.doi.org/10.1177/2049936114528173

4. Merchant $S$, Bharati A, Merchant N. Tuberculosis of the genitourinary system: Urinary tract tuberculosis: Renal tuberculosis-Part I. Indian J Radiol Imaging 2013;23:46-63. http://dx.doi.org/10.4103/09713026.113615

5. Merchant S, Bharati A, Merchant N. Tuberculosis of the genitourinary system: Urinary tract tuberculosis: Renal tuberculosis-Part II. Indian J Radiol Imaging 2013;23:64-77. http://dx.doi.org/10.4103/09713026.113617

6. Gibson MS, Puckett ML, Shelly ME. Renal tuberculosis. Radiographics 2004;24:251-6. http://dx.doi. org/10.1148/rg.241035071

7. Centers for Disease Control and Prevention: Trends in tuberculosis, 2013. http://www.cdc.gov/tb/ publications/factsheets/statistics/Trends2013.pdf. Accessed July 7, 2015

8. American Thoracic Society. Diagnostic standards and classification of tuberculosis in adults and children. Am J Respir Crit Care Med 2000;161:1376-95. http://dx.doi.org/10.1164/airccm.161.4.16141

9. Centers for Disease Control and Prevention. Treatment of tuberculosis, American Thoracic Society, CDC, and Infectious Diseases Society of America. MMWR 2003;52(№. RR-11).

10. Cek M, Lenk $S$, Naber KG, et al. EAU guidelines for the management of genitourinary tuberculosis. Eur Urol 2005;48:353-62. http://dx.doi.org/10.1016/i.eururo.2005.03.008

Correspondence: Dr. Sero Andonian, Associate Professor of Urology, Royal Victoria Hospital, McGill University Health Centre, 1001 boul. Decarie, Suite D05.5331, Montreal, Quebec, H4A 3J1; sero.andonian@muhc.mcgill.ca 